\title{
Ion Induced Lamellar-Lamellar Phase Transition in Charged Surfactant Systems
}

\author{
Daniel Harries, Rudi Podgornik ${ }^{\dagger}$ and V. Adrian Parsegian \\ Laboratory of Physical and Structural Biology, \\ National Institute of Child Health and Human Development, \\ National Institutes of Health, \\ Bethesda, Maryland 20892-0924, USA \\ and \\ $\dagger$ Faculty of Mathematics and Physics, \\ University of Ljubljana, \\ and Department of Theoretical Physics, \\ J. Stefan Institute, Ljubljana, Slovenia \\ Etay Mar-Or and David Andelman \\ School of Physics and Astronomy, Raymond and Beverly Sackler \\ Faculty of Exact Sciences, Tel Aviv University, Tel Aviv 69978, Israel
}

\begin{abstract}
We propose a model for the liquid-liquid $\left(\mathrm{L}_{\alpha} \rightarrow \mathrm{L}_{\alpha^{\prime}}\right)$ phase transition observed in osmotic pressure measurements of certain charged lamellae-forming amphiphiles. The model free energy combines mean-field electrostatic and phenomenological non-electrostatic interactions, while the number of dissociated counterions is treated as a variable degree of freedom that is determined self-consistently. The model, therefore, joins two well-known theories: the Poisson-Boltzmann theory for ionic solutions between charged lamellae, and Langmuir-Frumkin-Davies adsorption isotherm modified to account for charged adsorbing species. Minimizing the appropriate free energy for each interlamellar spacing, we find the ionic density profiles and the resulting osmotic pressure. While in the simple Poisson-Boltzmann theory the osmotic pressure isotherms are always smooth, we observe a discontinuous liquid-liquid phase transition when Poisson-Boltzmann theory is self-consistently augmented by Langmuir-Frumkin-Davies adsorption. This phase transition depends on the area per amphiphilic headgroup, as well as on non-electrostatic interactions of the counterions with the lamellae, and interactions between counterion-bound and counterion-dissociated surfactants. Coupling lateral phase transition in the bilayer plane with electrostatic interactions in the bulk, our results offer a qualitative explanation for the existence of the $\mathrm{L}_{\alpha} \rightarrow \mathrm{L}_{\alpha^{\prime}}$ phase-transition of DDABr (didodecyldimethylammonium bromide), but its apparent absence for the chloride and the iodide homologues. More quantitative comparisons with experiment require better understanding of the microscopic basis of the phenomenological model parameters.
\end{abstract}

\section{INTRODUCTION}

From naturally occurring phospholipids to synthetic double-chain surfactants, over a wide range of concentrations, amphiphiles in aqueous solutions self-assemble into multilamellar phases. The stability of the lamellar stack depends not only on the type of amphiphile, but also on the competition between several inter-lamellar interactions [1]. Attractive van der Waals interactions are balanced by repulsive interactions. Hydration repulsion usually dominates when the intervening water layer spacings are small (typically $\lesssim 1 \mathrm{~nm}$ ) or intermediate, while electrostatic and 'steric' undulation interactions usually prevail at intermediate to large spacing, up to hundreds of nanometers [2, 3, 4, 5, 6].

Between charged surfactants, the stabilizing repulsion is typically provided by the strong Coulomb interaction mediated by dissolved counterions and salt 7]. These interactions are particularly strong for lamellar-forming charged surfactants whose counterions fully dissociate into solution. Salt can attenuate such electrostatic interactions via ionic screening. For surfactants that form flexible layers, the complicated yet important coupling between layer elasticity, undulations, and electrostatic in- teractions must also be considered [4, 8, 9, 10]. But even when the effects of layer flexibility can be ignored, electrostatic interactions in multilamellar charged systems are non-trivial and, in general, difficult to understand because of the intimate link between counterion dissociation, ionic screening, and ion-specific non-electrostatic interactions 11, 12, 13.

The determination of the collapse pressure of membrane stacks by Dubois et al. 14 adds a new twist. Synthetic cationic double-chain surfactant, didodecyldimethylammonium $\left(\mathrm{DDA}^{+}\right)$with bromide as counterion (DDABr), is used to form a thermodynamically stable lamellar phase. The system undergoes a phase transition from a swollen liquid-like $\left(\mathrm{L}_{\alpha}\right)$ lamellar phase to another, more condensed, liquid-like lamellar phase $\left(\mathrm{L}_{\alpha^{\prime}}\right)$. This phase transition is induced by externally applied osmotic pressure; it is seen as a plateau in the osmotic pressure versus inter-lamellar spacing isotherms. Measured by small angle X-ray scattering, the abrupt change in spacing is typically between $10 \AA$ to $100 \AA$. In contrast, for the same surfactant with the bromide counterion replaced by chloride, $\mathrm{DDACl}$, there is no evidence of a first-order transition 14]. In fact, the experimental isotherm can be well fit by the usual Poisson-Boltzmann 
(PB) theory [4]. Further, with an iodide counterion the stack made of DDAI surfactant remains collapsed and does not swell at all 15. Remarkably, a discontinuous increase in area per surfactant with no discernible inplane positional order was experimentally found to coincide with the collapse in bilayer spacing. Clearly, this collapse is strongly coupled to a lateral rearrangement in the bilayer plane, and cannot be solely the result of neutralizing surfactant headgroups by their counterions.

It should perhaps come as no surprise that different halide counterions interact differently with the charged $\mathrm{DDA}^{+}$surfactant layers. The number of electrons, hence properties like polarizability, vary widely for these ions, and we expect that ion-membrane interactions will be different too. By ranking ions according to their efficiency in salting-out proteins from solution, Hofmeister was first to observe - over a century ago - that different ions partition differently at aqueous interfaces 16. The Hofmeister ranking is surprisingly insensitive to the details of the interface [17, 18, 19]. Often, however, the preferential interaction follows the size and polarizability of the ion; large ions tend to be less repelled from (or more attracted to) oily interfaces.

It has been proposed that the added van der Waals attraction of the ions to the higher index of refraction material may explain the Hofmeister ranking 20, 21, 22, 23, 24]. Water ordering around ions at the interface that is structured differently from the bulk can also discriminate between ions. More polarizable ions, for example, may be attracted to ordered water molecules at the interface because of favorable interaction between dipoles and induced-dipoles. Due to their amphiphilic, liquidlike nature, surfactants present a special and complex interface to water and salt ions. However, using measurements such as electrophoretic mobility, nuclear magnetic resonance (NMR), and buoyancy density-matching, a Hofmeister-like ranking of anions has also emerged for ions at lipid interfaces [25, 26, 27]. Also, for single chain micelle-forming cationic surfactants, the area per molecule follows the Hofmeister series, increasing more in the presence of larger ions. [28].

As is evident from NMR experiments, different ions not only associate differently with the amphiphile-water interface, but their binding may also restructure the interface itself 26]. Computer simulations indicate that the restructuring of the amphiphilic headgroup region should be strongly influenced by the counter-ion size [29]. Such conformational changes at the interface are possible sources of non-ideal lipid mixing, because ion binding at the interface may effectively create two incompatible types of lipids: ion-bound and ion-detached. For example, both experiments and simulations of lipid mixtures 30, 31, 32, 33, 34, 35. show that charged and uncharged lipids tend to demix so as to minimize the line tension between the different mismatched lipid species, often leading to lipid lateral phase separation in the membrane plane. Perhaps most compelling are the phase transitions from lamellar to inverted hexagonal phases of pure
DOPS induced by varying $\mathrm{pH}$ that changes the fraction of charged to uncharged ionizable lipids $[36]$.

Can an added non-electrostatic attraction of ions to the lipid-water interface explain the observed transition for $\mathrm{Br}^{-}$ions? The charge regulation model of Ninham and Parsegian 13 indicates that while an added attraction can significantly modify pressure isotherms, it cannot account for a first-order phase transition.

Here, we propose a phenomenological model that explains the first-order phase transition in terms of an added coupling between electrostatics and nonelectrostatic specific interactions at the interface. The model is motivated by the experimentally observed lamellar-lamellar phase transition in charged surfactant systems 14 and is a relatively simple extension of Poisson-Boltzmann theory.

The gist of our model is to consider the possibility that a fraction of the counterions are not dissociated from the lamellar-forming cationic $\mathrm{DDA}^{+}$surfactant, but rather stay associated with it on the membrane plane to form a neutral complex. The degree of dissociation is taken as a variational parameter in our free-energy formulation, and is optimized for each inter-lamellar distance 13]. Further, we consider each lamella as composed of a binary mixture of neutral (associated counterions) and charged (dissociated counterions) surfactant species. Assuming an effective attractive 2nd order virial coefficient between the two species, we find possible lateral phase separation in the lamellar plane forming neutralsurfactant rich and charged-surfactant rich phases, much as in regular solution theory [11, 37. Like any phenomenological model, our model relies on several parameters whose exact molecular origin is not well known at present. However, using reasonable values of these parameters, we are able to fit well the experimental data.

Our model couples the Poisson-Boltzmann theory for the counterions in solution with the Langmuir-FrumkinDavies adsorption model that regulates the amount of dissociated counterions [37, 38, 39, 40, 41. It is, therefore, an extension of the charge regulation model of Ninham and Parsegian [13]. Analogous coupling between surface transitions and bulk interactions has been analyzed in the context of hydration forces [42, 43] as well as electrostatic interactions 44 .

In the model, the differences between monovalent ions $\left(\mathrm{Cl}^{-}, \mathrm{Br}^{-}\right.$, and $\left.\mathrm{I}^{-}\right)$are accounted for by using different interaction parameters between ions and lipid interface and between ion-bound and ion-detached lipids. Together with the repulsive hydration force, known to act strongly at small separations such as those found in the collapsed phase, these interaction parameters are sufficient to reproduce the experimental observations. Our main emergent result supports a lamellar-lamellar phasetransition as function of externally applied osmotic pressure for ions such as $\mathrm{Br}^{-}$. At small osmotic pressure and large inter-lamellar distances, most of the $\mathrm{Br}^{-}$ions are dissociated, and the isotherm follows the PB result [40, 41]. However, for larger pressures and smaller 
separations, a large fraction of the $\mathrm{Br}^{-}$ions remain associated, causing a lateral phase transition. Because of the coupling between electrostatics and the entropy of ions in solution, the lateral phase transition also leads to the discontinuous jump in inter-lamellar spacing witnessed in the osmotic pressure isotherm.

We further consider the effect of added salt on the equilibrium state of the system. The main effect of salt is to screen electrostatic interactions, to reduce the coupling between the layers, and thus to diminish the magnitude of the first-order transition jump [45]. Indeed, our model predicts that for more than a critical amount of salt the phase transition disappears altogether.

The outline of the paper is as follows: in Sec. II we present an extension to the usual PB theory, taking into account the non-electrostatic degrees of freedom and treating separately the counterion-only and added-salt cases. In Sec. III we present our numerically calculated isotherms to show the possibility of a lamellar-lamellar phase transition. We then discuss the link with experiments and comment on ion-specific effects. In Sec. IV we discuss our findings and end in Sec. V with a short summary and remarks on possible future directions.

\section{EXTENDED POISSON-BOLTZMANN THEORY}

\section{A. Model}

The lamellar stack is composed of bilayers of doublechain surfactants such as DDA separated by regions of aqueous solution. The charged surfactant hydrophilic headgroups point towards the water region, while the hydrophobic tails are packed in the inner lamellar region, away from the polar water environment; for $\mathrm{DDABr}$, the thickness of the hydrocarbon part of the bilayer is of the order of $24-26 \AA$. The lamellar stack can be modeled as a one-dimensional periodic system. This approximates the lamellar lateral extent as infinite and each lamella as perfectly planar and rigid. We consider only the unit cell of the lamellar stack, as is depicted in Figure 1.

For convenience, the unit cell width $D$ spans only the aqueous inter-lamellar region, while the periodicity of the lamellar stack includes also the bilayer thickness: $D+D_{m}$. All local quantities depend only on the perpendicular coordinate, $z$. Because the solubility of single surfactants (like DDA) in water is extremely low, we assume that all surfactants reside within the lamellar bilayers. These bilayers are the two bounding interfaces of the unit cell.

The model system, hence, is composed of two charged interfaces separated by a distance $D$. The system is overall electroneutral; the amount of counterions in the aqueous region is exactly balanced by the amount of charged surfactant on the two interfaces. Note that because of the insolubility assumption, the ratio between the lamellar bilayer thickness and $D$ uniquely determines the relative

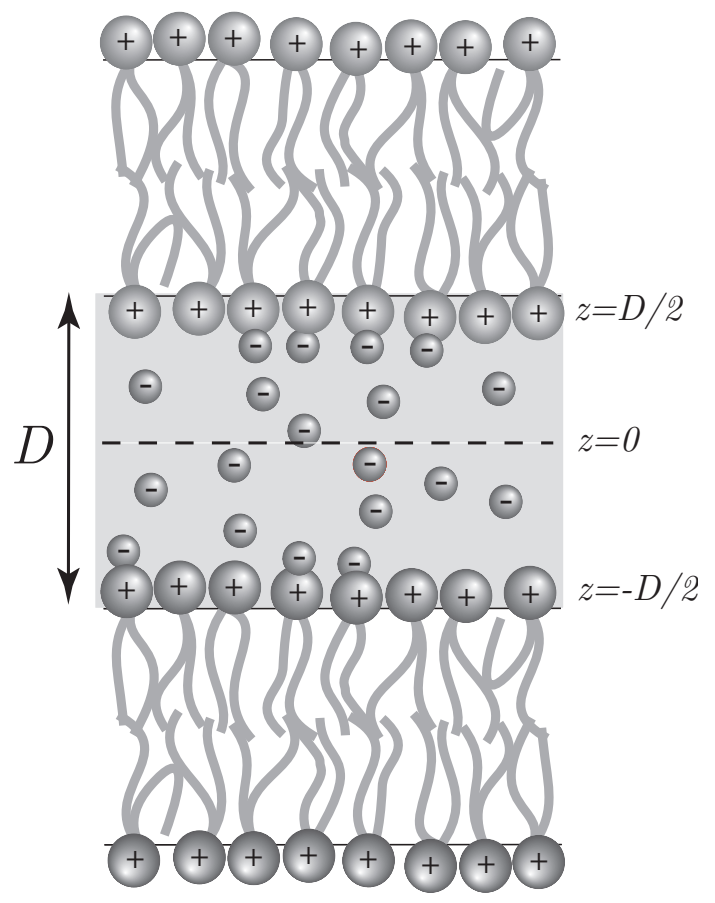

FIG. 1: A schematic view of the lamellar phase. The unit cell width includes only the water region, $-D / 2<z<D / 2$, and is composed of two charged surfaces located at $z= \pm D / 2$ (each being one leaflet of the surfactant bilayer). The counterions can adsorb on the two surfaces or dissociate into the inter-membrane water region of thickness $D$.

concentration of surfactant and water.

The midplane of the unit cell, see Figure 1, is chosen at $z=0$, from which the two interfaces bearing equal charge densities are located at $z= \pm D / 2$. Because the system is symmetric about the $z=0$ midplane, it is enough to consider a unit cell in the range $0 \leq z \leq$ $D / 2$. The counterion concentration (mole per liter) $c(\bar{z})$ and the mean-field electrostatic potential $\psi(z)$ depend on the perpendicular coordinate $z$. All variables calculated at the lamellar surface, $z=D / 2$, will be denoted by a subscript $s$ (e.g., $\psi_{s}$ ), while those calculated at the symmetric midplane, $z=0$, by a subscript $m$ (e.g., $\psi_{m}$ ).

Our aim is to calculate the equation of state for this lamellar symmetry and to express it as a relation between the thermodynamic variables: inter-lamellar distance, $D$, and osmotic pressure, П. Just as in the van der Waals phenomenological theory of phase transitions, we search for thermodynamically stable states or stable branches of the free energy. When we find more than one branch, we can use the Maxwell construction to obtain the coexistence region.

Minimization of the model free energy with respect to electrostatic and non-electrostatic degrees of freedom (see below) will eventually lead to the equation of state, our final goal. The overall free energy $F_{v}+F_{s}+F_{\text {hyd }}$ per unit cell area is a sum of volume contributions coming from the electrolyte solution within the cell, including ion (electrostatic) $F_{v}$ terms, surface contributions having their origin at the interfaces $F_{s}$, and hydration interac- 
tion $F_{\text {hyd }}$, dominant at small $D$ separations.

Because we approximate the hydration interaction as a separable term, we can independently minimize the electrostatic contribution to the free energy. As we show next, these interactions already suffice to account for a discontinues phase transition, while $F_{\text {hyd }}$ is needed only to account for the experimentally found pressure at small $D$. We therefore discuss only the minimization of $F_{\text {tot }}=F_{v}+F_{s}$ below, and return to present and discuss the added $F_{\text {hyd }}$ when we compare theory and experiment in the Results section.

Because the system is extensive in the interfacial area, the ion (electrostatic) volume free energy per unit area, $F_{v}$, is taken as the appropriate intensive quantity

$F_{v}=\int_{0}^{D / 2}\left[-e c \psi-\frac{\varepsilon}{8 \pi}\left(\psi^{\prime}\right)^{2}+k_{B} T c\left(\ln \left(c / c_{0}\right)-1\right)\right] \mathrm{d} z$.

The first two terms include the standard electrostatic energy, with $\varepsilon \simeq 80$ the dielectric constant of water. The last term is the entropy of mixing in the dilute limit, where $c_{0}$ is a reference concentration, $T$ the temperature, and $k_{B}$ the Boltzmann constant. Because all counterions in solution originate from a surfactant molecule, their integrated concentration (per unit area) must be equal in magnitude and opposite in sign to the surface charge density

$$
\sigma=-e \int_{0}^{D / 2} c(z) \mathrm{d} z
$$

This is also a charge density condition and can be translated via Gauss' law into the electrostatic boundary condition (in gaussian units): $\psi^{\prime}(D / 2)=\psi_{s}^{\prime}=4 \pi \sigma / \varepsilon$, linking the surface electric field $\psi_{s}^{\prime}$ with the surface charge density $\sigma$. Unlike the usual PB theory where either the surface charge or the surface potential is held fixed, here $\sigma$ is a self-adjusting parameter which will be determined variationally from minimizing the total free energy $F_{\text {tot }}$.

The second part of the total free energy comes from the surface free energy contributions of the amphiphiles residing on the planar bilayers. The surface free energy $F_{s}$ has electrostatic and non-electrostatic energy terms as well as a lateral mixing entropy contribution. Expressed in terms of the surface area fraction $\eta_{s}=a^{2} \sigma / e$ of charged surfactants,

$$
\begin{aligned}
a^{2} F_{s} & =e \psi_{s} \eta_{s}-\hat{\alpha} \eta_{s}-\frac{1}{2} \hat{\chi} \eta_{s}^{2} \\
& +k_{B} T\left[\eta_{s} \ln \eta_{s}+\left(1-\eta_{s}\right) \ln \left(1-\eta_{s}\right)\right]
\end{aligned}
$$

where the first term couples between the surface charge and surface potential. The other terms are the enthalpy and entropy of a two-component liquid mixture: charged surfactant with area fraction $\eta_{s}$ and neutralized, ionbound surfactants with area fraction $1-\eta_{s}$. The parameters $\hat{\alpha}$ and $\hat{\chi}$ are phenomenological, respectively describing the counterion-surfactant and the surfactantsurfactant interactions at the surface. As in the charge regulation model [13], here $\hat{\alpha}<0$ means that there is an added non-electrostatic attraction (favorable adsorption free energy) between counterions and the surface; the more counterions are associated at the surface, the smaller the amount of remaining charged surfactant.

The parameter $\hat{\chi}$ is the most crucial and unique element in our model. Representing non-ideal mixing tendencies in the bilayer plane as in regular solution theory, it alone (together with the usual components of standard PB theory) is sufficient to account for a coupled transition in the bilayer plane and in the bulk. As in the Frumkin adsorption model [37, 38, 39, 40, 41], a positive $\hat{\chi}$ parameter represents the tendency of surfactants on the surface to phase separate into domains of neutral and charged surfactants.

Changing to dimensionless variables, we define $y(z) \equiv$ $e \psi(z) / k_{B} T, \phi(z) \equiv a^{3} c(z), \alpha \equiv \hat{\alpha} / k_{B} T, \chi=\hat{\chi} / k_{B} T$, and take for convenience $c_{0} a^{3}=1$. Then, the total free energy is written as a functional of the variables $y(z)$, $\phi(z)$, and a function of $\eta_{s}$, and includes the conservation condition, eq 2$]$ via a Lagrange multiplier, $\mu$ :

$$
\begin{array}{r}
\frac{a^{2}}{k_{B} T} F_{\text {tot }}\left[y, \phi ; \eta_{s}\right]=\frac{a^{2}}{k_{B} T} F_{v}+\frac{a^{2}}{k_{B} T} F_{s} \\
-\mu\left[\eta_{s}-\frac{1}{a} \int_{0}^{D / 2} \phi(z) \mathrm{d} z\right] \\
=\frac{1}{a} \int_{0}^{D / 2}\left[-y(z) \phi(z)-\frac{a^{3}}{8 \pi l_{B}}\left(y^{\prime}\right)^{2}+\phi(\ln \phi-1)\right] \mathrm{d} z \\
+y_{s} \eta_{s}-\alpha \eta_{s}-\frac{1}{2} \chi \eta_{s}^{2}+\eta_{s} \ln \eta_{s}+\left(1-\eta_{s}\right) \ln \left(1-\eta_{s}\right) \\
-\mu\left[\eta_{s}-\frac{1}{a} \int_{0}^{D / 2} \phi(z) \mathrm{d} z\right]
\end{array}
$$

Next, we minimize $F_{\text {tot }}$ with respect to the surface variable $\eta_{s}$, and the two continuous fields $\phi(z), y(z)$ : $\mathrm{d} F_{\text {tot }} / \mathrm{d} \eta_{s}=\delta F_{\text {tot }} / \delta \phi(z)=\delta F_{\text {tot }} / \delta y(z)=0$, corresponding to three coupled equations of state

$$
\begin{aligned}
\frac{\eta_{s}}{1-\eta_{s}} & =\exp \left(\mu+\alpha+\chi \eta_{s}-y_{s}\right) \\
\phi(z) & =\exp (-\mu+y(z)) \\
y^{\prime \prime}(z) & =\frac{4 \pi e^{2}}{\varepsilon k_{B} T a^{3}} \phi(z)=\frac{4 \pi l_{B}}{a^{3}} \phi(z) .
\end{aligned}
$$

The free energy $F_{\text {tot }}$ is also a function of surface potential $y_{s}$ and of the inter-lamellar spacing $D$. The differentiation with respect to $D$ gives the osmotic pressure (to be discussed in Sec. II.B), while the variation with respect to $y_{s}$ gives the usual electrostatic boundary condition:

$$
y^{\prime}(D / 2)=y_{s}^{\prime}=\frac{4 \pi \eta_{s}}{a^{2}},
$$

where $l_{B}=e^{2} /\left(\varepsilon k_{B} T\right)$ is the Bjerrum length, equal to about $7 \AA$ at room temperature for aqueous solutions $(\varepsilon=80)$. The Lagrange multiplier, $\mu$, acts as a chemical potential, but with the important difference that it 
is related not to the bulk reservoir concentration, but rather to the concentration at the midplane, $\phi_{m}$. For a single counterion type, we can choose, without loss of generality, the potential at the mid-plane to be zero, $y_{m}=e \psi_{m} / k_{B} T=0$ and then from eqs 67

$$
\begin{aligned}
\phi(z) & =\phi_{m} \mathrm{e}^{y(z)} \\
\phi_{m} & =\mathrm{e}^{-\mu} \\
y^{\prime \prime}(z) & =\frac{4 \pi l_{B}}{a^{3}} \phi_{m} \mathrm{e}^{y(z)} .
\end{aligned}
$$

Not surprisingly, we recover the Poisson-Boltzmann equation 9 connecting the electrostatic potential $y(z)$ with the counterion concentration $\phi(z)$ in the solution. This can be expected since the non-electrostatic contributions enter only via the surface interactions expressed in eq 5 ,

Rewriting eq 5 we arrive at an expression similar to the Langmuir-Frumkin-Davies adsorption isotherm [39],

$$
\eta_{s}=\frac{1}{1+\phi_{m} \mathrm{e}^{-\alpha-\chi \eta_{s}+y_{s}}},
$$

with the following modifications: the concentration $\phi_{m}$ at the midplane replaces the bulk concentration (because of the constraint of overall charge neutrality), and the surface interaction energy in the exponent contains the electrostatic part $y_{s}$ 13]. The simpler Langmuir isotherm is recovered in the limit of non-charged surfaces, $y_{s}=0$, and no surface interaction, $\chi=0$ :

$$
\eta_{s}=\frac{1}{1+\phi_{m} \mathrm{e}^{-\alpha}} .
$$

Here, there is a unique relation between $\phi_{m}$ and $\eta_{s}$, while in the general case of non-zero $y_{s}$ and $\chi$, the generalized Langmuir-Frumkin-Davies equation offers a transcendental relation between $\eta_{s}, \phi_{m}$ and $y_{s}$ with the possibility of more than one solution.

The solution of the PB equation 9 for two symmetric charged surfaces separated by a distance $D$, each having a surface charge density of $\sigma$, has a well-known analytic form [4, 13, 46]

$$
y(z)=-\ln \left[\cos ^{2}(K z)\right],
$$

where the constant $K$ is determined from the electrostatic boundary condition $y^{\prime}(D / 2)=y_{s}^{\prime}=4 \pi l_{B} \eta_{s} / a^{2}$, eq 8 as

$$
K D \tan (K D / 2)=\frac{2 \pi l_{B}}{a^{2}} \eta_{s} D .
$$

We can now express $y_{s}$ and $\phi_{m}$ as function of a single dimensionless variable $u \equiv K D / 2$ :

$$
\begin{aligned}
y_{s} & =-\ln \left[\cos ^{2}(u)\right] \\
\eta_{s} & =\frac{C_{1}}{D} u \tan (u) \\
\phi_{m} & =\frac{C_{2}}{D^{2}} u^{2},
\end{aligned}
$$

with the constants $C_{1}$ and $C_{2}$ obtained as

$$
\begin{aligned}
& C_{1}=\frac{a^{2}}{\pi l_{B}} \\
& C_{2}=\frac{2 a^{3}}{\pi l_{B}}=2 a C_{1} .
\end{aligned}
$$

The solution of the above equations, together with the adsorption isotherm eq [10 completely determines the counterion density profile and the mean electrostatic potential via the solution $K=K(D, \alpha, \chi)$.

\section{B. Equation of State and the $\mathrm{L}_{\alpha} \rightarrow \mathrm{L}_{\alpha^{\prime}}$ Phase Transition}

We now solve the basic set of equations derived in the previous section. Substituting $\phi_{m}, \eta_{s}$, and $y_{s}$ into the Langmuir-Frumkin-Davies isotherm, eq [10] we get an equation for $u$

$$
\frac{D}{C_{1} u \tan (u)}=1+\frac{C_{2}}{\cos ^{2}(u)}\left(\frac{u}{D}\right)^{2} \exp \left(-\alpha-\chi \frac{C_{1}}{D} u \tan (u)\right) \text {. }
$$

Obviously, for each imposed distance $D$, we can extract $u=K D / 2$ from eq [16] Once $u$ is known, it can be substituted into eq 14 wherefrom $\eta_{s}, \phi_{m}, y_{s}$ follow. Their values completely determine the potential profile $\psi(z)=k_{B} T y(z) / e$ and counterion profile $c(z)=\phi(z) / a^{3}$.

If there is only one solution for $u$, the system has one stable state. Multiple $u$ solutions indicate the possibility of coexistence between several phases, as well as transitions between them. Each phase corresponds to a separate branch of the free energy with its own dependence on $D$. If all solutions of eq [16 are non-zero, then we can have a first-order transition between two stable phases.

The free energy as a function of $D$ and $\eta_{s}(D)$ can be obtained by substituting the results of minimization back into eq 4 yielding

$$
\frac{a^{2}}{k_{B} T} F_{\text {tot }}=-\frac{1}{2 \pi l_{B}}\left[4 \pi l_{B} \eta_{s}-\frac{1}{2}(K a)^{2} D\right]+\frac{1}{2} \chi \eta_{s}^{2}+\ln \left(1-\eta_{s}\right) \text {. }
$$

The appropriate isotherm is now obtained by taking the derivative of the free energy with respect to $D$, giving the osmotic pressure $\Pi(D)$ as

$$
\Pi(D)=-\frac{\mathrm{dF}_{\mathrm{tot}}}{\mathrm{d} D} .
$$

As is usual in PB theory [4], the osmotic pressure can also be calculated from the contact theorem, which relates the osmotic pressure with the value of the counterion concentration at the interface. Counterion concentration at the interface $(z=D / 2)$ can be, in turn, connected with the concentration at the midplane $(z=0)$, thus yielding an alternative form of the osmotic pressure as

$$
\Pi(D)=k_{B} T c_{m} .
$$


This latter equation has exactly the same form as in the standard PB theory. The only way non-electrostatic terms of the free energy influence the osmotic pressure is via the solution of eq 16 Both forms of the osmotic pressure, eqs 18 and [19] of course yield exactly the same values.

A typical isotherm $\Pi(D)$ (in Pascal units) calculated using eqs 16] and 19 is shown in Figure 2a, and the corresponding surface charge density in Figure $2 \mathrm{~b}$. Note that is this example we do not include contributions from hydration. We discuss the parameter range in the next section, and choose the parameters here to be $\alpha=-6$, $a=8 \AA$ and $\chi=12$. The isotherm clearly exhibits a first-order phase transition from one free energy branch at large inter-lamellar separation $D$ to another at smaller $D$, and with a coexistence region in between. For large values of $D, D \geq 64 \AA$, most counterions are dissociated from surfactants, $\eta_{s} \lesssim 1$, and the osmotic pressure follows the standard PB theory for (almost) fully dissociated surfactants. For $D$ smaller than $39 \AA, \eta_{s} \leq 0.1$, and the isotherm follows another branch, characterized by a much smaller surface charge of only about $10 \%$ of the fully dissociated value. For $39 \AA \leq D \leq 64 \AA$ the system exhibits a coexistence between two phases. The pressure has a plateau and $\eta_{s}$ changes from one branch to the second.

The plateau in the osmotic pressure between the two stable solutions on Figure 2a is evaluated using the usual Maxwell construction. This plateau in $\Pi(D)$ indicates a first-order phase transition between the solutions corresponding to two stable branches of the free energy: a condensed one with $D \simeq 39 \AA$ and a dilute one with $D \simeq 64 \AA$. Although it is hard to fit the phenomenological parameters $\alpha, a$ and $\chi$ to the DDABr results of Ref. [14], we believe that the mechanism proposed here and the typical results presented in Figure 2 are relevant to the experimental system. More details are given in Sec. III below, where we show how the phase transition depends on model parameters.

\section{Added-Salt Case}

After demonstrating the possibility of a phase transition for the counterions only, we generalize the results by coupling the lamellar system to a reservoir of 1:1 monovalent salt. This generalization is easily achieved within our model. The main effect of the salt is to diminish or even to eliminate completely the phase transition.

The main modifications of the model wrought by the introduction of salt ions are as follows. First we must consider ionic concentration profiles independently for positive and negative ions: $\phi^{+}(z)$ and $\phi^{-}(\mathrm{z})$. Note that we do not distinguish between the negative salt counterions and those dissociated from the surfactant. The free energy is written, similarly to eq 4 in terms of rescaled variables with the introduction of two chemical potentials. The first, $\mu^{+}$, is coupled to the excess amount of co-ions with
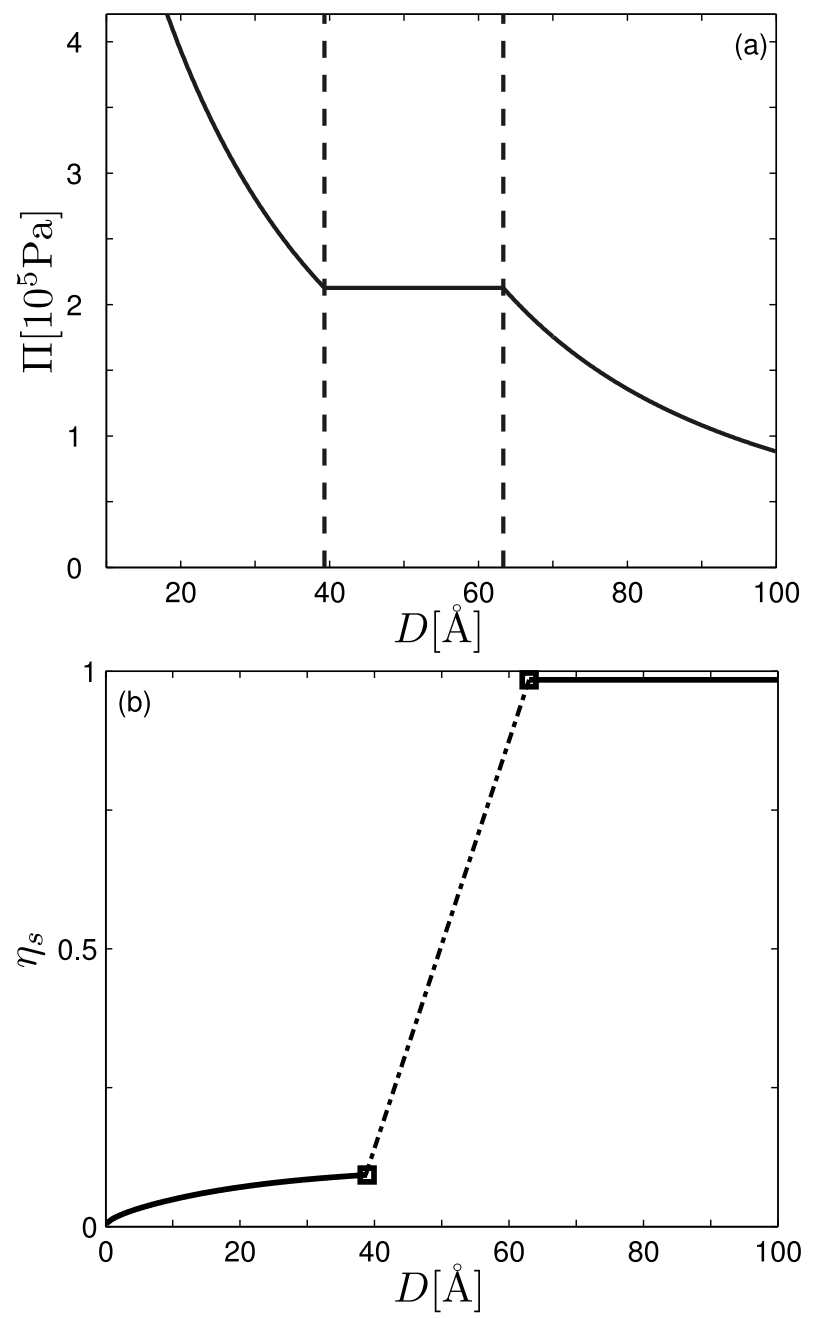

FIG. 2: (a) The osmotic pressure $\Pi$ in units of $10^{5}$ Pascals $(\approx 1 \mathrm{~atm})$; and, (b) the area fraction $\eta_{s}=a^{2} \sigma / e$ of surface charges, as function of inter-lamellar spacing $D$ for $\alpha=-6, \chi=12$ and $a=8 \AA$. The Maxwell construction gives a coexistence between a phase with $D \simeq$ $39 \AA$ and low $\eta_{s} \leq 0.1$, and another with $D \simeq 64 \AA$ and $\eta_{s} \approx 1$. In (b) the two coexisting phases are denoted by squares and the dotteddashed line shows the tie-line in the coexistence region.

respect to the reservoir, stemming from the integral of $\phi^{+}$ in the region between the two plates. The second, $\mu^{-}$, is coupled with the excess number of counterions, stemming from the integral of $\phi^{-}$in the region between the two plates with the added contribution of surface charges 
$1-\eta_{s}$. Thus, the total free energy is given by

$$
\begin{aligned}
& \frac{a^{2}}{k_{B} T} F_{\text {tot }}\left[y, \phi ; \eta_{s}\right]=\frac{a^{2}}{k_{B} T} F_{v}+\frac{a^{2}}{k_{B} T} F_{s} \\
+ & \mu^{+}\left[\frac{1}{a} \int_{0}^{D / 2}\left[\phi^{+}(z)-\phi_{0}^{+}\right] \mathrm{d} z\right] \\
+ & \mu^{-}\left[\frac{1}{a} \int_{0}^{D / 2}\left[\phi^{-}(z)-\phi_{0}^{-}\right] \mathrm{d} z+\left(1-\eta_{s}\right)\right] \\
= & \frac{1}{a} \int_{0}^{D / 2}\left[-y(z)\left[\phi^{-}(z)-\phi^{+}(z)\right]\right. \\
- & \left.\frac{a^{3}}{8 \pi l_{B}}\left(y^{\prime}\right)^{2}+\phi^{-}\left(\ln \phi^{-}-1\right)+\phi^{+}\left(\ln \phi^{+}-1\right)\right] \mathrm{d} z \\
+ & y_{s} \eta_{s}-\alpha \eta_{s}-\frac{1}{2} \chi \eta_{s}^{2}+\eta_{s} \ln \eta_{s}+\left(1-\eta_{s}\right) \ln \left(1-\eta_{s}\right) \\
+ & \mu^{+}\left[\frac{1}{a} \int_{0}^{D / 2}\left[\phi^{+}(z)-\phi_{0}^{+}\right] \mathrm{d} z\right] \\
+ & \mu^{-}\left[\frac{1}{a} \int_{0}^{D / 2}\left[\phi^{-}(z)-\phi_{0}^{-}\right] \mathrm{d} z+\left(1-\eta_{s}\right)\right] . \quad(20)
\end{aligned}
$$

Taking now the variation of the above free energy with respect to $\eta_{s}, \phi^{ \pm}$and $y$, we get the Euler-Lagrange equations,

$$
\begin{aligned}
\frac{\eta_{s}}{1-\eta_{s}} & =\exp \left(\mu^{-}+\alpha+\chi \eta_{s}-y_{s}\right) \\
\phi^{-}(z) & =\exp \left(-\mu^{-}+y(z)\right) \\
\phi^{+}(z) & =\exp \left(-\mu^{+}-y(z)\right) \\
y^{\prime \prime}(z) & =\frac{4 \pi e^{2}}{\varepsilon k_{B} T a^{3}}\left(\phi^{-}(z)-\phi^{+}(z)\right) \\
& =\frac{4 \pi l_{B}}{a^{3}}\left(\phi^{-}(z)-\phi^{+}(z)\right) .
\end{aligned}
$$

The final variation with respect to $y_{s}$ gives the usual electrostatic boundary condition relating the surface electric field with the surface charge density: $y_{s}^{\prime}=\left(4 \pi l_{B} / a^{2}\right) \eta_{s}$, eq 8 Requiring that the bulk concentration of co-ions and counterions matches the reservoir concentration, $\phi_{0}$, where the potential vanishes, $y=0$, it is easily verified from eqs 2224 that

$$
\begin{aligned}
\mu^{ \pm} & =-\ln \phi_{0} \\
\phi^{ \pm}(z) & =\phi_{0} \mathrm{e}^{\mp y(z)} \\
y^{\prime \prime}(z) & =\frac{8 \pi l_{B} \phi_{0}}{a^{3}} \sinh (y(z))=\lambda_{D}^{-2} \sinh y,
\end{aligned}
$$

where

$$
\lambda_{D}=\left(\frac{8 \pi l_{B} \phi_{0}}{a^{3}}\right)^{-1 / 2}
$$

is the Debye-Hückel screening length. Inserting eq25into 21 leads to a Langmuir-Frumkin-Davis isotherm, now of the form:

$$
\eta_{s}=\frac{1}{1+\phi_{0} \mathrm{e}^{-\alpha-\chi \eta_{s}+y_{s}}} .
$$

This equation resembles eq 10, only that in eq 29] the reservoir concentration $\phi_{0}$ takes the place of $\phi_{m}$. Moreover, the value of the potential at the midplane is not fixed, but rather is determined from $\phi_{0}$, as are the concentrations, $\phi_{m}^{ \pm}=\phi_{0} \exp \left(\mp y_{m}\right)$.

The PB equation in presence of salt, eq 27 depends on the electrostatic boundary conditions and has a wellknown solution expressed via an elliptic integral [4]. The first integration of the PB equation, eq27 from the midplane position $(z=0)$ to an arbitrary $z$ gives

$$
y^{\prime}(z)=\frac{1}{\lambda_{D}} \sqrt{2 \cosh y(z)-2 \cosh y_{m}} .
$$

The boundary condition can be inserted in eq[30] yielding

$$
\cosh y_{s}=\cosh y_{m}+\frac{\pi l_{B}}{a \phi_{0}} \eta_{s}^{2}
$$

A further integration can be written in terms of an elliptic function

$$
\frac{z}{\lambda_{D}}=\int_{y_{m}}^{y} \frac{\mathrm{d} w}{\sqrt{2 \cosh w-2 \cosh y_{m}}},
$$

and the second boundary condition $y_{s}=y(z=D / 2)$ can be expressed as

$$
\frac{D}{2 \lambda_{D}}=\int_{y_{m}}^{y_{s}} \frac{\mathrm{d} w}{\sqrt{2 \cosh w-2 \cosh y_{m}}} .
$$

The procedure to solve these equations is similar to the one used in the previous section. For given $D, \phi_{0}, \alpha$, $\chi$ and $a$, the profiles $\phi^{ \pm}(z)$ and the surface value $\eta_{s}$ are calculated numerically and inserted into the free energy expression, eq [20] $F_{\text {tot }}\left[\phi^{ \pm}(z) ; \eta_{s}, D\right]$. Taking the derivative of $F_{\text {tot }}$ with respect to $D$, or equivalently using the contact theorem as for the counterion-only system, the osmotic pressure, $\Pi(D)$ is obtained,

$$
\Pi(D)=k_{B} T\left(c_{m}^{+}+c_{m}^{-}-2 c_{0}\right)=\frac{k_{B} T}{a^{3}}\left(\phi_{m}^{+}+\phi_{m}^{-}-2 \phi_{0}\right) .
$$

\section{RESULTS}

To better appreciate the role of ionic species in determining the phase transition we first present results for counterions-only and in the absence of hydration interactions. These results will already clearly show the most important feature of the model, namely the possible lamellar-lamellar transition. We then predict the effect of added salt. Finally, we include the hydration contribution and compare model results with experiments.

\section{A. Ion Dependent Lamellar-Lamellar Transition}

Our model contains three parameters: $\alpha, \chi$ and $a$. For $a$ we use the linear size of the surfactant headgroup, typically in the range of $7 \AA$ to $9 \AA$ with cross-sectional area 


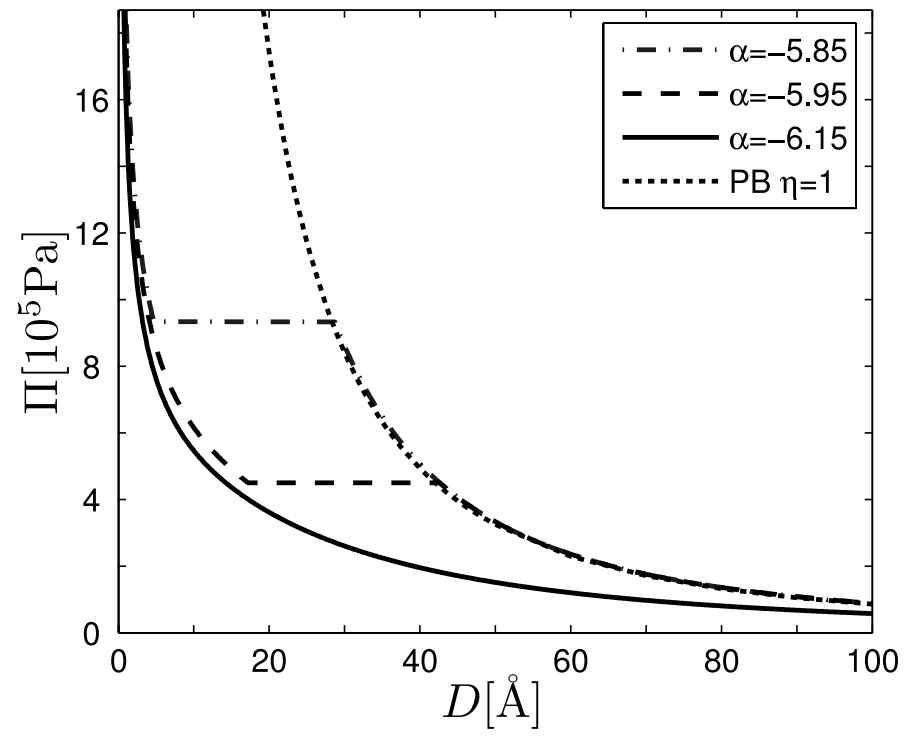

FIG. 3: The osmotic pressure isotherm $\Pi(D)$ for three binding strengths: $\alpha=-5.85$ (dotted-dashes), -5.95 (dashed line), and -6.15 (solid line). The other parameters are for non-ideal mixing $\chi=12$ and lateral separation $a=8 \AA$. The $\alpha=-5.85$ and -5.95 lines show a phase transition, while $\alpha=-6.15$ does not show one. For comparison, the usual PB isotherm with $\eta_{s}=1$ (short dashes) is also shown.

$a^{2}$. For the phenomenological constants $\alpha$ and, in particular, for $\chi$ there is no direct and accurate experimental measurement. However, estimates consistent with experimental data (as discussed in Secs. IIIC and IV), will be used hereafter. In our model, positive $\chi$ presents the possibility of lateral phase separation. In addition, for $\alpha<0$, the counterions tend to stay associated with the charged headgroup and reduce the surface charge density.

In Figure 3 we compare the osmotic pressure isotherm $\Pi(D)$ in the case of no added salt, for three $\alpha$ values (and for a constant $\chi=12$ and $a=8 \AA$ ) with the standard $\mathrm{PB}$ isotherm (short dashes), for the fully dissociated limiting case, $\eta_{s}=1$. Formally, full dissociation can be achieved by setting $\alpha \rightarrow \infty$ in our equations. For the two values of $\alpha=-5.85,-5.95$, the isotherms in Figure 3 show a firstorder phase-transition in the range of $10 \AA \lesssim D \lesssim 50 \AA$. The phase transition is from a dilute and highly charged $\mathrm{L}_{\alpha}$ lamellar phase (large $D$ and $\eta_{s} \lesssim 1$ ), to another $\mathrm{L}_{\alpha^{\prime}}$ phase that is more condensed and less charged (small $D$ and $\eta_{s} \ll 1$ ). As $\alpha$ increases, this phase transition shifts to higher values of $\Pi$ and lower values of $D$.

For large $D, \eta_{s}$ is very close to one, and the osmotic pressure isotherm closely follows the $\mathrm{PB}$ result; for large $D$ the solution of our model remains essentially on the $\mathrm{PB}$ branch of the free energy, characterized by $\eta_{s} \simeq 1$. For small values of inter-lamellar spacing, $D$, the values of osmotic pressure for all three values of $\alpha$ are again very similar, with small and slowly varying $\eta_{s}$, as in Figure $2 \mathrm{~b}$. Here, the system essentially remains on the associated branch of the free energy characterized by $\eta_{s} \ll 1$.

The lowest $\alpha$ isotherm (solid line, $\alpha=-6.15$ ) shows no

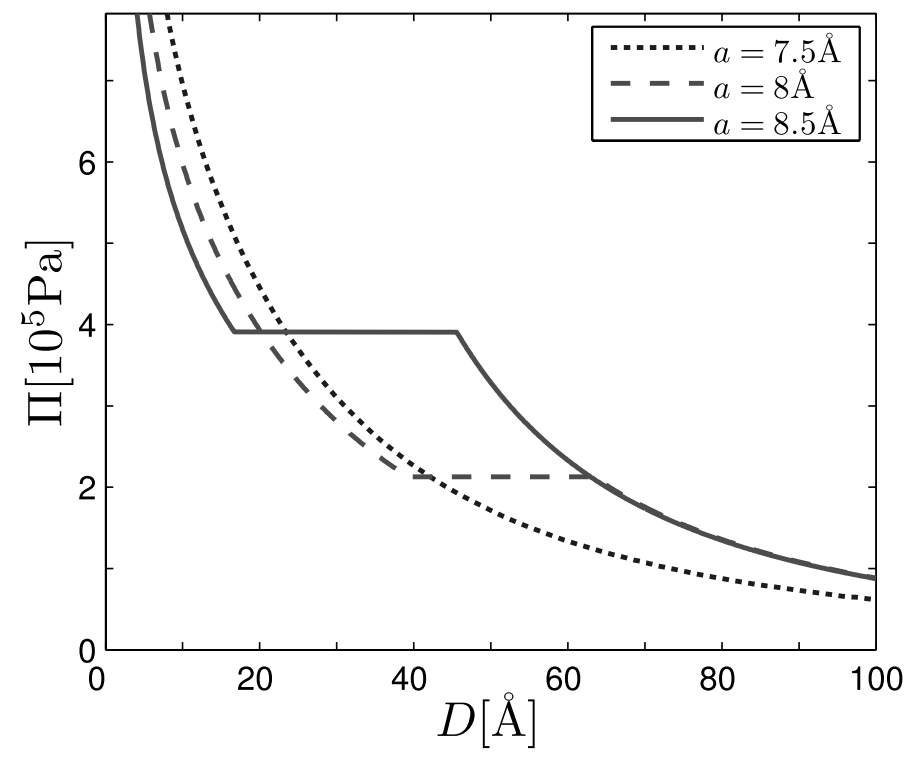

FIG. 4: The osmotic pressure isotherm $\Pi(D)$ for three values of the surfactant headgroup separation: $a=7.5 \AA$ (short-dashes), $8 \AA$ (dashed line), and $8.5 \AA$ (solid line). The other parameters are $\alpha=-6$ and $\chi=12$. A phase transition is seen for $a=8 \AA$ and $8.5 \AA$, but not for $a=7.5 \AA$.

transition. The counterions are almost fully associated in this case for the entire range of $D$, leading to a lower value of pressure for all $D$ 's. Note that all three osmotic pressure isotherms as well as the $\mathrm{PB}$ one have the same limiting behavior for $D \rightarrow \infty$. This is quite accurately described by the Langmuir form of the osmotic pressure, as applied to the counterion-only case [4]:

$$
\Pi(D)=\frac{k_{B} T \pi}{2 l_{B}} \frac{1}{D^{2}}
$$

which does not depend on the value of the surface change (or, equivalently on $\eta_{s}$ ).

In Figures 4 and 5 we show the effects of the variation of linear size $a$ and lateral interaction $\chi$, respectively. We have chosen the parameter range to show isotherms without a phase transition (small $a$ or $\chi$ ) as well as those showing the transition (higher values of $a$ or $\chi$ ) in each of the figures. The main features are the same as in Figure 3. The large $D$ region represents highly dissociated lamellae (strongly charged), while the phase transition (when it exists) can be seen for small $D$ at higher lamellar density. Increasing $a$ moves the transition point towards higher values of osmotic pressure or equivalently lower values of $D$ until it eventually disappears. Increasing $\chi$ has the same effect. Note also that the pressure at low $D$ is diminished on increase of $a$. This effect can be understood by recalling the relation $\sigma=e \eta_{s} / a^{2}$, so that for the same $\eta_{s}$, larger $a$ corresponds to smaller surface charge density $\sigma$.

Our results are summarized in Figure 6 where the $(\alpha$, $\chi)$ parameter space is separated by a solid descending line $\chi_{c}(\alpha)$ into two regions (for fixed $a$ ). The upper region (large $\chi$, large $\alpha$ ) corresponds to isotherms with a 


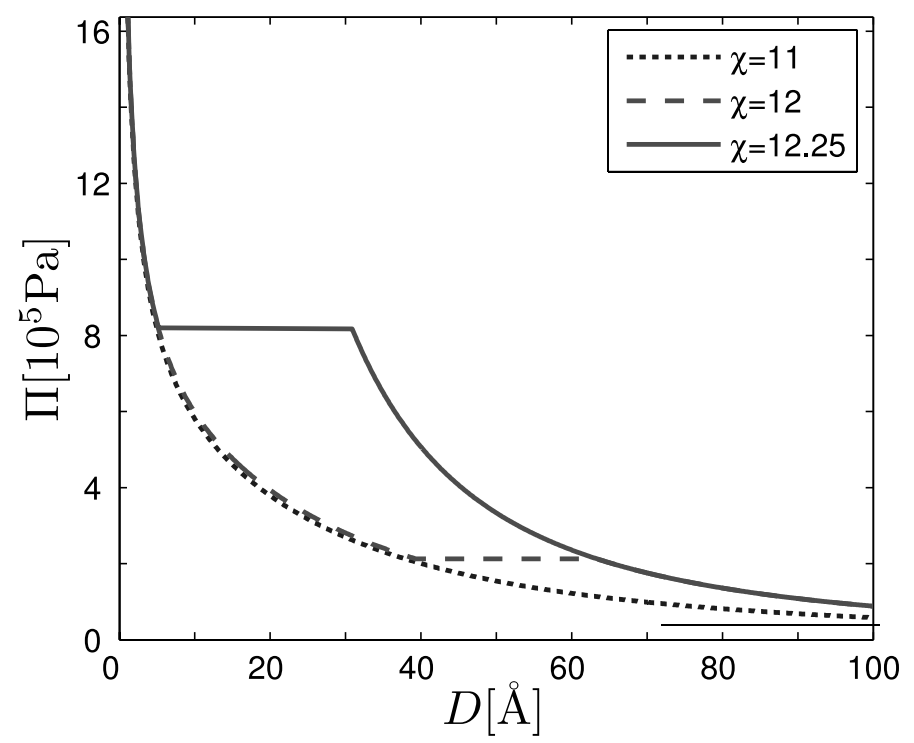

FIG. 5: The osmotic pressure isotherm $\Pi(D)$ for three $\chi$ values: $\chi=11$ (short dashes), 12 (dashed line), and 12.25 (solid line). The other parameters are $\alpha=-6$ and $a=8 \AA$. The phase transition is seen for the two larger values of $\chi$.

phase transition (and is designated as "two phases" on the figure). Below that region (small $\chi$, small $\alpha$ ) the isotherms show no phase transition (designated as "one phase" on the figure). The degree of counterion dissociation varies in this region from very small values to values $\eta_{s} \lesssim 0.8$ for finite values of $\alpha$ and $\chi$. The PB result of $\eta_{s}=1$ is reached only asymptotically as $\alpha \rightarrow \infty$. The line represents the continuous line of critical point in the $(\chi, \alpha)$ plane. The region between the solid and dot-dashed lines corresponds to jumps in $D$ at the transition of more than $\approx 3 \AA$. Above the dot-dashed line, the behavior at $D \gtrsim 3 \AA$ is described by the usual PB solution because the transition occurs at unphysically small $D$ values.

The inset to Figure 6 corresponds to variations of $\chi$ and $\alpha$ shown in Figure 4 and 5, respectively. As $\chi$ or $\alpha$ are lowered, the single-phase region is eventually reached. Figure 6 is plotted for $a=8 \AA$. As $a$ decreases, the twophase region shrinks and the one-phase region expands.

The dashed line in the figure is an analytic calculation which gives the following approximate form of $\chi^{*}(\alpha)$

$$
\chi^{*} \simeq-2 \alpha+2 \ln \left(\frac{2 \pi l_{B}}{a}\right)-4 .
$$

To derive this result we assume that the phase transition occurs at large $D$. Using the asymptotic large $D$ behavior, we compare the free energy of $\eta_{s} \simeq 1$ with $\eta_{s} \ll 1$ and determine the transition point as a function of $\chi$ for given $\alpha$ and $a$. As can be seen by comparing the analytic (dashed) line with the full numerical solution (solid line), the approximation is good for small $\alpha<0$. For $\alpha \gtrsim-3$ the assumption of a transition at large $D$ breaks down and the approximated $\chi^{*}$ starts to deviate considerably from the numerically calculated $\chi_{c}(\alpha)$.

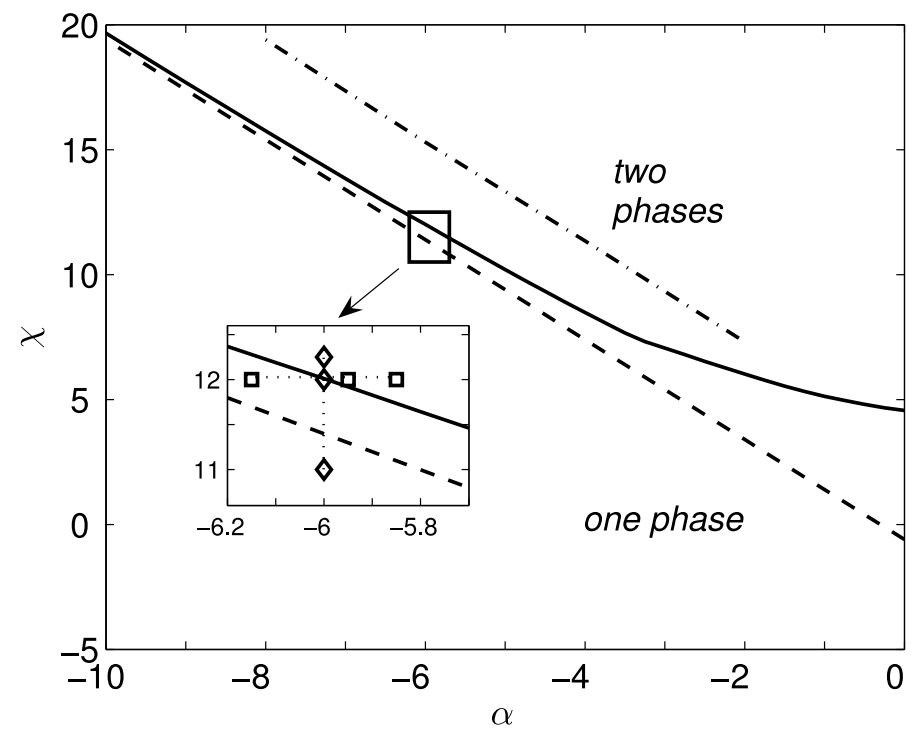

FIG. 6: Phase diagram in the $(\alpha, \chi)$ plane for $a=8 \AA$. In the region below the solid line $\chi_{c}(\alpha)$, the system does not exhibit a phase transition ("one phase"). The dashed line is the analytical approximation of $\chi^{*} \simeq-2 \alpha+2 \ln \left(2 \pi l_{B} / a\right)-4$. The inset is a blow-up of the region around $\alpha \approx-6$ and $\chi \approx 12$, showing the parameters used in figures 3 and 5. Square symbols correspond to isotherms in Figure 3, while diamonds correspond to those in Figure 5. The region lying between the full and dot-dashed lines in the two-phase region corresponds to transitions in $\mathrm{D}$ that are larger than $\approx 3 \AA$.

\section{B. Added Salt: Vanishing of the Transition}

The effect of added salt was treated in Sec. IIc. The salt is characterized by the Debye-Hückel screening length $\lambda_{D}$, and screens electrostatic interactions. As the amount of added salt increases, $\lambda_{D}$ decreases, and the phase transition observed in the absence of salt becomes gradually less pronounced until it is finally wiped out completely [45]. This is clearly seen in Figure 7. In 7a, three osmotic pressure isotherms are shown. A plateau (first-order phase transition) is seen for the two lower amounts of salt, $c_{b}=10$ and $30 \mathrm{mM}$, while the transition disappears for higher amounts of salt, $c_{b}=50 \mathrm{mM}$.

One can also see how the phase transition is first shifted towards the high $D$ low $\Pi$ values, and then (for $\left.c_{b} \approx 40 \mathrm{mM}\right)$ completely disappears. The overall decrease in $\Pi(D)$ as the amount of salt increases, is due to the increased screening, and is present also in the simple $\mathrm{PB}$ theory. In $7 \mathrm{~b}$ the jump in $\eta_{s}$ is shown for $c_{b}=10$ and $30 \mathrm{mM}$, while it vanishes for higher amounts of salt, $c_{b}=50 \mathrm{mM}$, in accord with the isotherm behavior.

It is instructive to follow the change of the transition pressure $\Pi_{\text {tr }}$ as salt is added to the system, which is related to the difference in volume $\Delta V$ and number of ions $\Delta N$ in the two phases in a Clausius-Clapeyron-like equation:

$$
\frac{d \Pi_{\mathrm{tr}}}{d c_{b}}=\frac{k_{B} T}{c_{b}} \frac{\Delta N}{\Delta V} .
$$

Remarkably, we find an almost linear dependence of $\Pi_{\mathrm{tr}}$ 

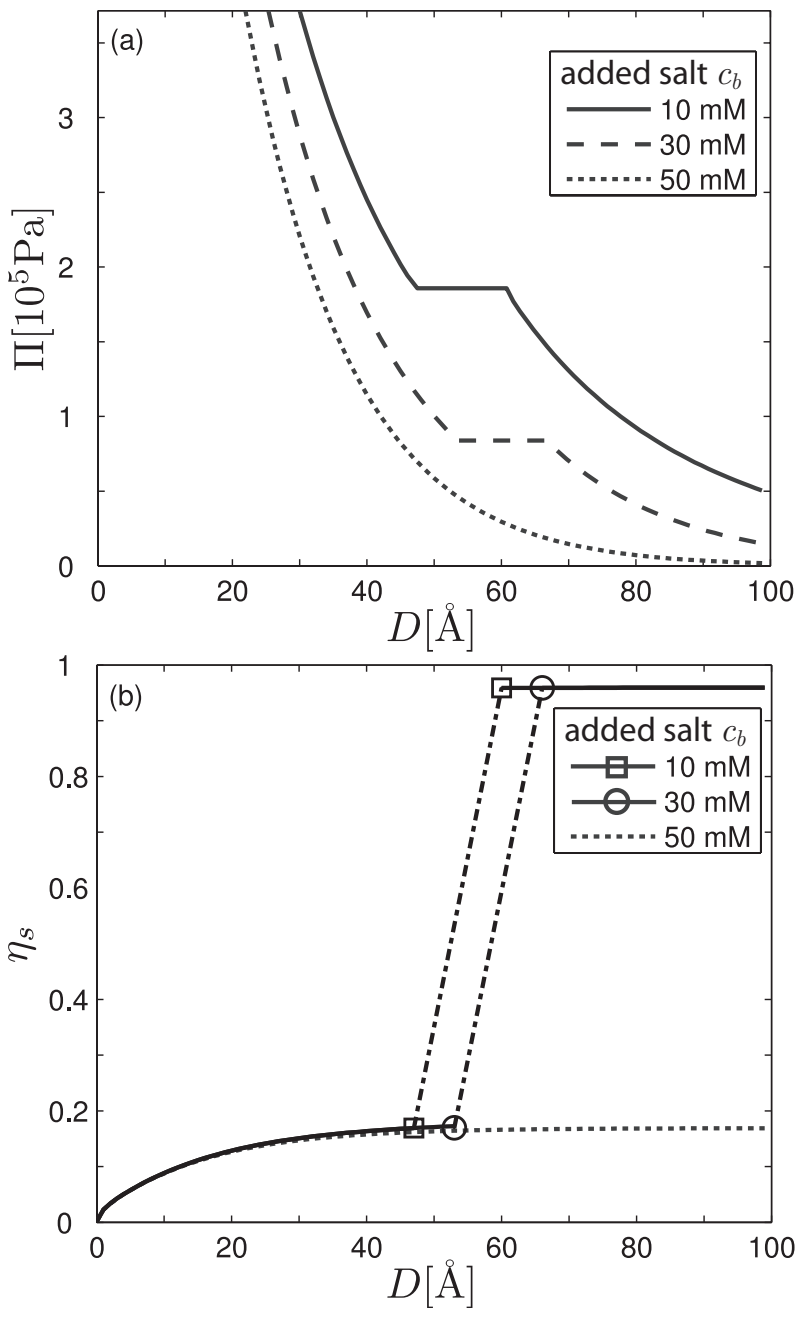

FIG. 7: Effect of added salt. In (a) $\Pi(D)$, in (b) $\eta_{s}(D)$ are plotted as a function of added salt concentration $c_{b}$. In (a) and (b): $c_{b}=10 \mathrm{mM}$ (solid line), $30 \mathrm{mM}$ (dashed line), $50 \mathrm{mM}$ (short dashes). In (c) salt concentration varies continuously over the range of existence of the transition, $0 \leq c_{b} \leq 40 \mathrm{mM}$. Other parameters are $\alpha=-5, \chi=10.19$, and $a=8 \AA$. As $c_{b}$ increases, screening becomes more important. The entire osmotic pressure isotherm $\Pi(D)$ decreases in magnitude and the phase transition region diminishes and shifts towards higher $D$ values. Note that for highest salt concentration, $c_{b}=50 \mathrm{mM}$, the phase transition has vanished. In (b) the coexisting values of the two phases are denoted by a square $\left(c_{b}=10 \mathrm{mM}\right)$ and by a circle $\left(c_{b}=30 \mathrm{mM}\right)$. The dotted-dashed lines are the corresponding tie-lines.

in the whole range of $c_{b}$, starting with the transition pressure at no added salt and leading eventually to the loss of transition for sufficiently high salt concentrations, $c_{b} \approx 40 \mathrm{mM}$, see figure 8 . In eq $37 d \Pi_{\mathrm{tr}} / d c_{b}$ corresponds to an added work of $2 k_{B} T$ due to the exclusion of ions acting on the volume change at the transition.

It is also interesting to follow the change in $\Pi_{t r}$ with temperature. Experimentally, both an increase of $\Pi_{\mathrm{tr}}$ with $T$ (at lower $T$ ) and a decrease (at higher $T$ ) have been observed [14]. In contrast, if we assume that $\chi$ and $\alpha$ are independent of $T$ in the model, we find that $\Pi_{\mathrm{tr}}$ monotonically, almost linearly, increases with $T$. This

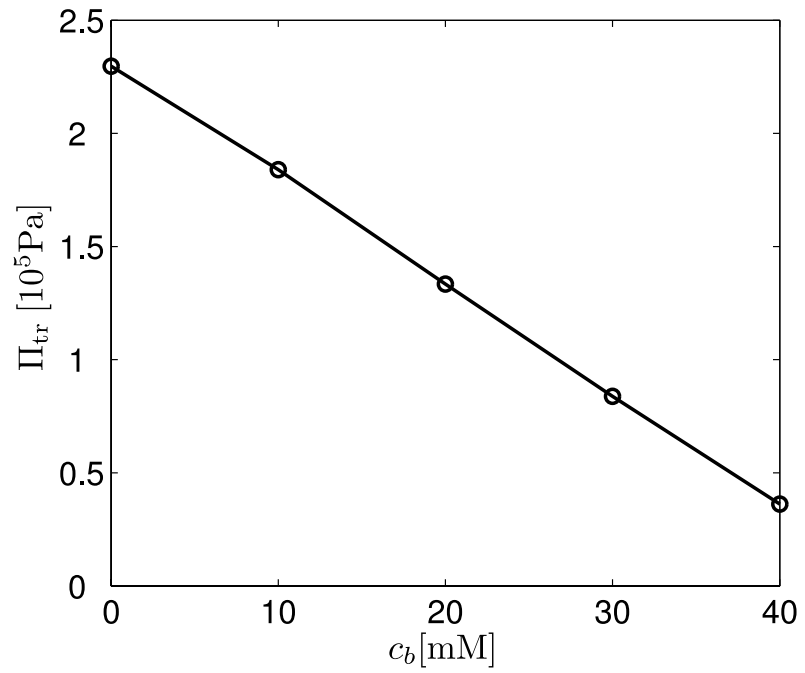

FIG. 8: Effect of added salt on the transition pressure. $\Pi_{\operatorname{tr}}\left(c_{b}\right)$ is plotted as a function of added salt concentration $c_{b}$. The dependence of the transition pressure on the salt concentration in the region where the transition exists is linear, with a slope of $7.9 \times 10^{-21} \mathrm{~J}$.

suggests that in a more refined model, the parameters $\chi$ and $\alpha$ should be taken as functions of the temperature rather than simple constants. For example, if we assume that $\alpha(T)$ and $\chi(T)$ vary as $1 / T$, while the ratio $\alpha / \chi$ is kept constant, we find that $\Pi_{\mathrm{tr}}$ decreases monotonically with $T$.

\section{Relating to DDABr/DDACl Osmotic Pressure Experiments}

The experimentally observed difference between $\mathrm{DDABr}$ and DDACl for the different halides can easily be rationalized within our model by different values of $\alpha$ and/or $\chi$, for the different ions. This is indeed reasonable since experiments show that larger halide ions have an added affinity even for neutral lipids 25, 26, 27, 28]. The tendency of ions to preferentially partition into the hydrocarbon-water interface is most often reported in terms of an effective binding interaction that acts in addition to the repulsive electrostatic force, expected for ions interacting with low dielectric material. These differences in binding affinity would translate into a different value of $\alpha$ within our model. In these terms, experiments show that $|\alpha|$ is larger for bromide by one to four $k_{B} T$ more than for chloride, and iodide is at least an order of magnitude larger than those 25, 26, 27, 28].

With our model assumptions, we can now try to fit the experimental data in Ref. 14] using the same (small) amount of added salt as in the experiment, i.e. $c_{b}=$ $0.5 \mathrm{mM}$. The fit to the DDABr and DDACl lamellar systems are shown in Figure 9. We will first address the fit to the simpler case of DDACl that does not show in experiments a liquid-liquid coexistence, and then discuss $\mathrm{DDABr}$, where the liquid-liquid coexistence is clearly dis- 

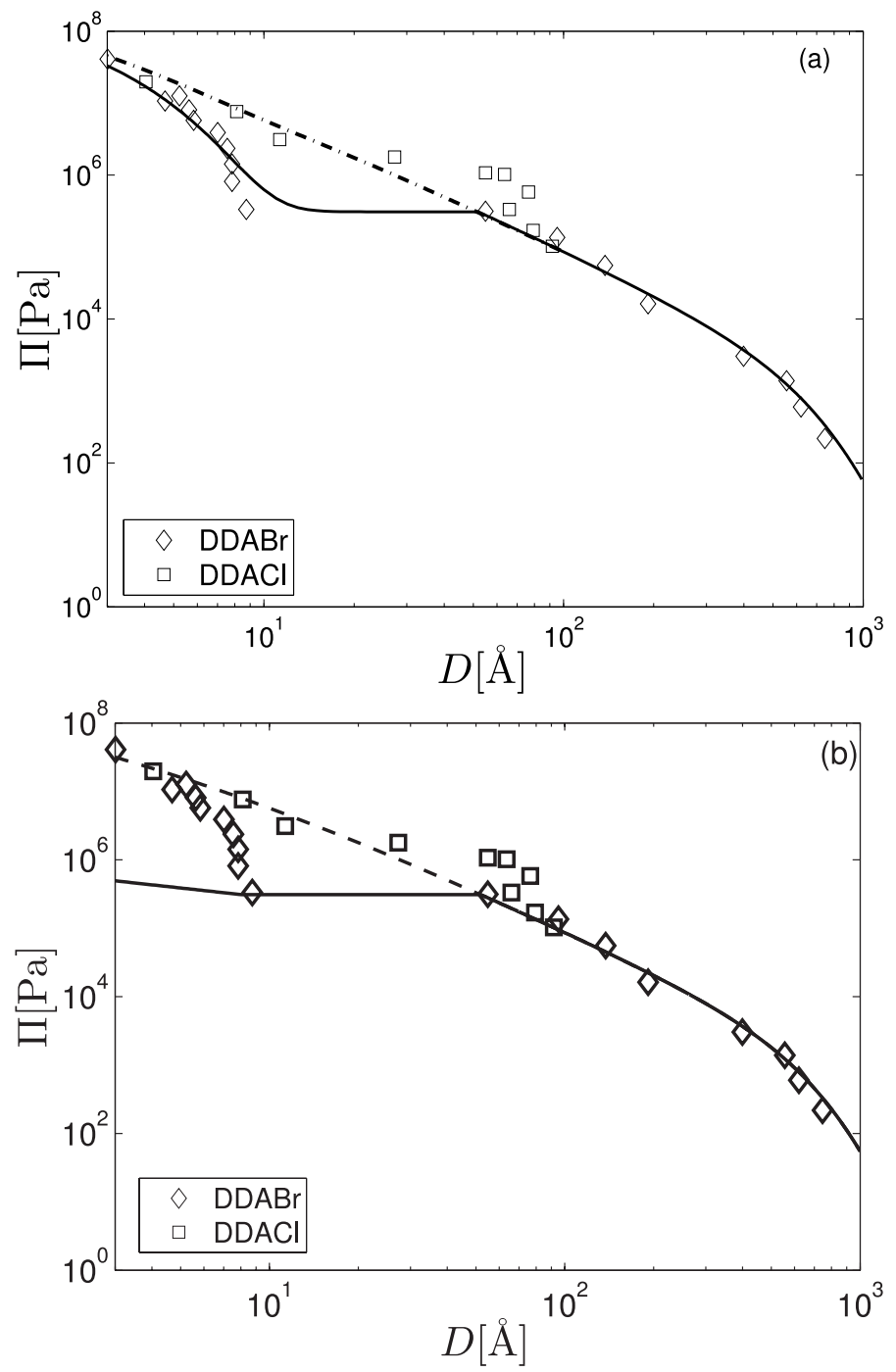

FIG. 9: Fit to the experimental osmotic pressure isotherm $\Pi(D)$ of Ref. 14] on a log-log scale. The diamonds and squares are the data points for DDABr and DDACl, respectively, reproduced from Ref. 14]. In (a) the solid line is the best fit of the model to the phase transition seen for DDABr with $\alpha=-7.4, \chi=14.75$ and $a=8 \AA$. The fit also includes a hydration contribution (parameters for the form: $\Pi_{\text {hyd }}=\Pi_{0} \exp \left(-D / \lambda_{\text {hyd }}\right)$, with typical values: $\Pi_{0}=2.37 \cdot 10^{8} \mathrm{~Pa}$ and $\lambda_{\text {hyd }}=1.51 \AA$ ). This contribution is particularly important at the low $D$ region of the DDABr isotherm. A small amount of salt is added in the fit as in the experiment $\left(c_{b}=0.5 \mathrm{mM}\right)$. The dot-dashed line is the fit to the DDACl (no transition). All parameters here are the same, except $\alpha=-3.4$ (one-phase region of Figure 6 ). In (b) the same model fits, showing only the electrostatic contributions to the force, $F_{v}+F_{s}$, that are responsible for the apparent phase transition. The fit to both data sets is good. However, the steep increase in pressure of the $\mathrm{DDABr}$ seen in the low $D$ range $(D<10 \AA)$ cannot be reproduced with these forces alone.

cerned. The DDACl data was fitted in Figure 9a using $\alpha=-3.4, \chi=14.75$ and $a=8 \AA$. The DDACl data points, represented by squares, are reproduced from Ref. 14]. The value chosen for $\chi$ is higher, yet close to $\chi_{c}(\alpha)$ (See Figure 6).

In comparing to experiments, it is important to realize that for small spacings, interactions of non-electrostatic origin tend to dominate the osmotic equilibrium [3, 5, 14, 47, 48]. Even for highly charged systems, hydration interactions acting at very short range, practically independent of the charging equilibrium at the surface, invariably dominate at separations of $D \lesssim 10 \AA$ are certainly among the most important to consider. Therefore, we add the hydration interaction $F_{\text {hyd }}$ as an idealized separable term to the overall interaction energy: $F_{\text {tot }}+F_{\text {hyd }}=F_{v}+F_{s}+F_{\text {hyd }}$. In conformity with hydration interactions between lipids 49] and between other macromolecules [4], we use the phenomenological form of an exponential interaction with a salt-independent decay length, $\lambda_{\text {hyd }} 50$

$$
F_{\text {hyd }}=\Pi_{0} \lambda_{\text {hyd }} \exp \left(-D / \lambda_{\text {hyd }}\right) .
$$

To account for the hydration interaction, we fit with $\Pi_{0}=$ $2.37 \times 10^{8} \mathrm{~Pa}$ and $\lambda_{\text {hyd }}=1.51 \AA$. These values are similar to those found from fits to experimental inter-lamellar spacings of lipids 14, 47, 51.

Note that our extended PB model predicts a transition at these values of $\alpha$ and $\chi$. However, this phase transition occurs at very small inter-lamellar separations of $D \lesssim 3 \AA$, indicating that only at such low $D$ values are the ions associated (small $\eta_{s}$ ). For higher $D$ values, the ions dissociate and the model follows the regular PB osmotic pressure. Values of $D \lesssim 3 \AA$ are well below the validity limit of the model; for such low $D$ 's the computed osmotic pressure isotherm can not be considered realistic. For higher $D$, the surface charge density, $\eta_{s}$, is almost one and the system exhibits the usual Poisson-Boltzmann isotherm, with screened electrostatic interactions at large $D$. We remark that the fit in the high $D$ region is very good, and surprisingly, also persists to small $D$ (although there are very few data points below $D=30-40 \AA$ ).

The DDABr was fitted in Figure 9a using a different $\alpha=-7.4$ but the same $\chi=14.75$ and $a=8 \AA$, as well as the same hydration interaction. The value of $\chi$ was picked near $\chi_{c}$ so that the transition would occur at a value of $D$ in the range of 10 to $40 \AA$. In this range our model is still valid and nicely reproduces a coexistence between the two liquid lamellar phases $\mathrm{L}_{\alpha}$ and $\mathrm{L}_{\alpha^{\prime}}$.

To better appreciate the role of hydration, figure $9 \mathrm{~b}$ shows the model fits that exclude the hydration contribution to the free energy. At smaller separations, $D<10 \AA$, the fit deviates considerably from the experimental osmotic pressure isotherm. While electrostatic forces can account for the transition point itself, they can definitely not by themselves reproduce the sharp rise in the osmotic pressure in the undissociated branch following the phase transition, compare Figure 9a.

Juxtaposing Figures $9 \mathrm{a}$ and $9 \mathrm{~b}$, it is evident that adding the hydration contribution affects mainly the undissociated branch at small inter-surface separations, since at large spacings electrostatic $\mathrm{PB}$ contributions completely overwhelm the much shorter ranged hydration. The hydration interaction mainly affects the small $D$ region of both $\mathrm{DDACl}$ and $\mathrm{DDABr}$. In fact, while 
the magnitude of hydration forces may not differ greatly from other (electrostatic) acting forces, the rate of force decay (slope) at such low spacings indicates that hydration is always acting. One can observe this in the context of lipids [48] as well as DNA [52]. Fits to both DDACl and DDABr should, therefore, contain hydration contributions to the free energy.

Importantly, as can be realized from Figure 6, there are different ways to cross the transition line, $\chi_{c}(\alpha)$, to witness the liquid-liquid coexistence. Another possible choice of parameters could thus assume the variation in $\chi$ instead of $\alpha$. For example, the data can be equally well fit if we fix $\alpha=-7.4$ and fit the DDABr data with $\chi=14.75$, and the DDACl with $\chi=18$.

Finally, we address the iodide analog, DDAI, that in experiments appears not to form a swollen liquid phase, remaining in the condensed liquid lamellar phase for the whole range of osmotic pressure values [15]. In the language of our model DDAI has either large $|\alpha|$ or $\chi$ values; it never enters the PB branch but instead remains in the undissociated branch for all spacings $D$. This corresponds to the "one phase" region of figure 6. Indeed, $|\alpha|$ for iodide could be estimated as yet larger than in the case of DDABr, probably by up to an order of magnitude [25, 28]. The system is confined to remain on the undissociated branch for this counterion: the repulsion due to electrostatic interactions and entropy of counterions is not strong enough to combat the (van der Waals) attractions. These attractions that are strong enough to hold the system in the secondary free energy minimum, effectively prevent the swelling observed for the other two counterions. We could thus establish a ranking in the model's parameter space, where DDACl, DDABr and DDAI would make a monotonic Hofmeister-like series either in the values of $|\alpha|$ or $\chi$. The 3rd parameter, $a$, is not changed in the fit because it is taken as the size of the same $\mathrm{DDA}^{+}$headgroup.

\section{DISCUSSION}

The model presented in this paper combines electrostatic and non-electrostatic interactions between charged surfactant bilayers. The non-electrostatic part of the interactions is accounted for using two phenomenological parameters characterizing the strength of the counterionamphiphile interaction, $\alpha$, and lateral amphiphileamphiphile interactions, $\chi$, on the charged dissociable surface. By choosing $\alpha$ to be negative, corresponding to favorable adsorption energy, and $\chi$ to be positive, corresponding to a net attractive interactions between likespecies, promoting lateral segregation between the dissociated and non-dissociated surfactants on the surface, we are able to qualitatively explain the experimentally observed $\mathrm{L}_{\alpha} \rightarrow \mathrm{L}_{\alpha^{\prime}}$ lamellar-lamellar phase transition.

\section{A. Origin of Phase Transition}

An abundance of experimental verification 25, 26, 27, 28] indicates that the different halides interact differently with lipids, forcing us to recognize the existence of nonelectrostatic interactions at work. In our model, this preferential interaction is represented by $\alpha$. By reducing the effective layer charge density, a favorable preferential interaction of ions to the interface acts to lower the pressure at any given spacing $D$. However, $\alpha$ alone cannot account for the abrupt jump in $D$ associated with a first-order phase transition. In fact, in the absence of $\chi$, pressure isotherms for any value of $\alpha$ are smooth (no phase transition).

Our model offers a natural extension of the PB theory with the Langmuir-Frumkin-Davies adsorption theory as applied to simple counterions. The large difference in behavior of the halide ions is modeled by the parameters $\alpha, \chi$ and $a$. The interaction parameters $\alpha$ and $\chi$ necessarily involve contributions from changes in hydration, solvation and desolvation, of lipid headgroups and their counterions. The model, therefore, underscores the important role of water structuring around ions devolved in the bulk versus at the interface.

The salient feature of our model is the first-order transition in the osmotic pressure isotherm from an almost completely dissociated state (highly charged and swollen, PB branch) at large interlayer separations, to an almost neutral, weakly dissociated, state approaching bilayer contact (condensed, undissociated branch). The existence of this transition depends on the values of both phenomenological parameters, but it is present over an extended region of phase space. The PB branch of the osmotic pressure isotherm is not much different from the standard PB theory with complete dissociation, both with or without added salt. On the other hand, the undissociated branch is characterized by a large attenuation in the magnitude of the osmotic pressure for a given inter-lamellar spacing, being about two orders of magnitude smaller then in the PB case.

In our model the $\mathrm{L}_{\alpha} \rightarrow \mathrm{L}_{\alpha^{\prime}}$ transition in the interlamellar spacing is coupled to a lateral first-order phase transition of the $\eta_{s}$ order parameter. This is a direct consequence of the coupling between inter-lamellar electrostatic degrees of freedom (mean electrostatic potential, mean ion density) and the surface non-electrostatic degrees of freedom as quantified by the phenomenological parameters $\alpha$ and $\chi$. The ensuing liquid-liquid lamellar phase transition is thus not only from one state of the lamellae where a larger fraction of the amphiphiles are charged to another state where they are less charged, but also from a state where the inter-lamellar forces are by and large electrostatic in nature, to a state where they are dominated by hydration. While in many experimental systems this transition is smooth and gradual, it is quite pronounced and discontinuous in the system studied here and in Ref. [14]. 


\section{B. Relating Model Parameters and Molecular Interactions}

We propose that non-ideal mixing between counterionassociated and dissociated surfactants can be responsible for an in-plane transition, which, in turn, is coupled to the bulk transition. This proposed non-ideality is represented in our model by $\chi$, as is sometimes used to report on lipids showing phase transitions following changes in $\mathrm{pH}$ [30]. While at present direct experimental verification and estimates for the proper $\chi$ values are lacking, we propose that conformational changes of lipid headgroups, ions and water structuring induced by the adsorbing ion, together with an added van der Waals interaction between neutralized surfactant complexes can lead to significant demixing. Furthermore, because larger ions are expected to perturb the lipid-water interface to a greater extent, it is reasonable to expect that the value of $\chi$ will follow a similar ranking to the binding of ions to the interface, represented by $\alpha$. We note, that the $\chi$ values needed to observe a phase transition, typically $\approx 10 k_{B} T$, are quite high. These high values are needed to overcome the electrostatic repulsion between like-charged lipids in this unscreened, highly charged system. The source of this lipid demixing energy (our $\chi$ parameter) could be associated with mismatch of headgroup-headgroup interactions, such as hydrogen bonding between neutral lipids, water-structuring forces, or non-electrostatic ionmediated interactions between lipids across two apposed bilayers for small inter-lamellar separations.

The parameter $a^{2}$ models the area per headgroup on the membrane plane. It is a function of several molecular interactions and, in principle, can be determined variationally. In Ref. [14] the area/headgroup was found to vary in a non-trivial fashion, from a larger value in the condensed lamellae to a smaller one in the dilute lamellae. The forces determining the area per surfactant are as yet unknown. Therefore, in the model we have not allowed for changes in area per surfactant, but note that it is not inconsistent to assume that the area per headgroup differs for the neutral vs. charged surfactant. The expansion of lipid area upon condensation, contrary to what is typically observed in phase transitions of lipids, could be evidence for direct attraction between $\mathrm{Br}^{-}$ions and the lipid hydrocarbon core, as suggested previously by Ninham et al 17]. This point deserves further investigation. Interestingly, however, as seen in Figure 4, the model predicts that lipids with larger area per headgroup can show a larger transition gap, as was found experimentally in the case of GM-3 ganglioside with weakly adsorbing counterions [53].

Another point that merits further investigation is the dependence of the isotherms on temperature. Both $\alpha(T)$ and $\chi(T)$ are complex functions of the temperature, with specific dependence that cannot be obtained from our model. A change in temperature affects the values of $\alpha(T)$ and $\chi(T)$ and can change the plateau pressure values, as was measured and reported in Figure 9 of
Ref. 14]. A better understanding of $\alpha(T)$ and $\chi(T)$ may offer an explanation to the non-monotonic behavior of the plateau pressure as function of temperature 14].

Finally we point out that the lack of direct experimental evidence at this time, particularly for $\chi$, limits out predictions to be mainly qualitative. More specifically, we can offer only a qualitative explanation for the strong difference in the behavior for different counterions, namely: no transition, transition and no stable swollen lamellar phase for the DDACl, DDABr and DDAI amphiphilic systems, respectively. Therefore, the fit to the data points shown on Figure 8 should be regarded as a tentative explanation of the mechanism behind the observed phase transition. For example, we cannot establish whether the main difference in ionic interactions with the surface are properly characterized by the value of $\alpha$ as opposed to $\chi$.

\section{CONCLUDING REMARKS}

Previously, phase transitions in DDABr lamellar systems have been theoretically attributed to either an ion-dependent van der Waals attraction between layers 6, 54], or to a strong-coupling effect between adsorbed ions, expected for surfaces with high charge density [55]. Here, we have shown that it is possible to account for the phase transition assuming a non-electrostatic interaction between ion-dissociated and ion-bound surfactants. We suggest that this interaction is ion specific and, hence, we offer an explanation for the different behavior seen for the three halide counterions.

The large phenomenological parameters we have found in our own fits of the data (see Fig. 9) as well as the large energetic terms assumed in the other approaches 6, 54, 55], all indicate that substantial attraction necessarily acts to overcome the electrostatic repulsion between surfactants. The molecular origin of this large energy has not yet been determined, and further experimental verification of the different phenomenological parameters is required. It will also be of interest to see if the lateral phase transition underlying the swelling transition and its dynamical evolution from one lamellar state to the other, can be observed directly in experiments.

We hope that in follow-up studies, a more microscopic approach will be able to shed light on the origin of the phase transition in these charged lamellar systems and how they relate to specific molecular details.

\section{Acknowledgements}

We are indebted to Th. Zemb for numerous comments and suggestions. We benefited from discussions with L. Belloni, H. Diamant, M. Dubois, and H.I. Petrache. D.A. acknowledges the hospitality of the LPSB/NICHD (NIH), where this work was completed and support from the U.S.-Israel Binational Science Foundation (B.S.F.) 
under grant No. 287/02, and the Israel Science Foundation under grant No. 160/05. This research was supported in part by the Intramural Research Program of the NIH, NICHD.
[1] V. A. Parsegian, Langmuir 9, 3625 (1993).

[2] W. Helfrich, Naturforsch. 33a, 305 (1978).

[3] E. A. Evans, and V. A. Parsegian, Proc. Natl. Acad. Sci. (USA) 837132 (1986).

[4] D. Andelman, in Handbook of Biological Physics: Structure and Dynamics of Membranes", Vol. 1B, edited by Lipowsky R.; Sackmann E. (Elsevier Science B.V., Amsterdam, 1995), ch. 12.

[5] H. I. Petrache, N. Gouliaev, S. Tristram-Nagle, R. Zhang, R. M. Suter, and J. F. Nagle, Phys. Rev. E 57, 7014 (1998).

[6] Th. Zemb, L. Belloni, M. Dubois, and S. Marcelja, Prog. Coll. Polym. Sci. 89, 33 (1992).

[7] C. Holm, P. Kékicheff, and R. Podgornik, (Editors), Electrostatic Effects in Soft Matter and Biophysics ( Kluwer,Dordrecht, 2001).

[8] C. R. Safinya, E. B. Sirota, D. Roux, and G. S. Smith, Phys. Rev. Lett. 62, 1134 (1989).

[9] G. D. Guttman, and D. Andelman, J. Phys. II (France) 3, 1411 (1993).

[10] D. Harries, S. May, and A. Ben-Shaul, J. Phys. Chem. B 107, 3624 (2003).

[11] S. May, D. Harries, and A. Ben-Shaul, Phys. Rev. Lett. 89268102 (2002).

[12] K. Arnold, in Handbook of Biological Physics: Structure and Dynamics of Membranes, Vol. 1B, edited by R. Lipowsky R.; Sackmann, E. (Elsevier Science B.V., Amsterdam, 1995) ch. 19.

[13] B. W. Ninham, and V. A. Parsegian, J. Theor. Biol. 31, 405 (1971).

[14] M. Dubois, Th. Zemb, N. Fuller, R. P. Rand, and V. A. Parsegian, J. Chem. Phys. 108, 7855 (1998).

[15] Zemb, Th. personal communication.

[16] F. Hofmeister, Archiv. Exp. Path. Pharm. 24, 247 (1887).

[17] P. Kunz, P. L. Nostro, and B. Ninham, Curr. Opin. Colloid Interface Sci. 9, 1 (2004).

[18] K. Collins, and M. Washbaugh, Q. Rev. Biophys. 18, 323 (1985).

[19] B. C. Garrett, Science 303, 1146 (2004).

[20] B. W. Ninham, and V. Yaminsky, Langmuir 13, 2097 (1997).

[21] P. Attard, D. J. Mitchell, and B. W. Ninham, J. Chem. Phys. 88, 4987 (1988).

[22] A. Becheri, P. L. Nostro, B. W. Ninham, and P. Baglioni, J. Phys. Chem. B 107, 3979 (2003).

[23] P. Jungwirth, and D. J. Tobias, J. Phys. Chem. B 106, 6361 (2002).

[24] M. C. Gurau, S.-M. Lim, E. T. Castellana, F. Albertorio, S. Kataoka, and P. S. Cremer, J. Am. Chem. Soc. 126, 10522 (2004).

[25] S. A. Tatulian, Biochim. Biophys. Acta 736189 (1992).

[26] J. R. Rydall, and P. M. Macdonald, Biochemistry 31, 1092 (1992).

[27] H. I. Petrache, I. Kimchi, D. Harries, and V. A. Parsegian, J. Am. Chem. Soc. 127, 11546 (2005).

[28] Th. Zemb, L. Belloni, M. Dubois, A. Aroti, and E. Leon- tidis, Curr. Opin. Coll. Int. Sci. 9, 74 (2004).

[29] J. N. Sachs, and T. B. Woolf, J. Am. Chem. Soc. 125, 8742 (2003).

[30] P. Garidel, C. Johann, and A. Blume, J. Lip. Res. 10, $131(2000)$.

[31] M. G. Noro, and W. M. Gelbart, J. Phys. Chem. 111, 3733 (1999).

[32] J. Nardi, R. Bruinsma, and E. Sackmann, Phys. Rev. E 58, 6340 (1998).

[33] J. Huang, and G. W. Feigenson, Biophys. J. 65, 1788 (1993).

[34] J. Huang, J. E. Swanson, A. R. G. Dibble, A. K. Hinderliter, and G. W. Feigenson, Biophys. J. 64, 413 (1993).

[35] B. Pozo Navas, K. Lohner, G. Deutsch, E. Sevcsik, K. A. Riske, R. Dimova, P. Garidel, G. Pabst, Biochim Biophys. Acta 1716, 40 (2005).

[36] N. Fuller, C. R. Benatti, and R. P. Rand, Biophys. J. 85, 1667 (2003).

[37] Hill, T. L. Introduction to Statistical Thermodynamics (Addison-Wesley, New-York, 1960).

[38] A. W. Adamson, and A. P. Gast Physical Chemistry of Surfaces (Wiley and Sons, New-York, 1997), Chap. XI, XVI.

[39] J. T. Davies, Proc. Royal Soc. A 245, 417 (1958).

[40] H. Diamant H., and D. Andelman, J. Phys. Chem. 100 13732 (1996); ibid. Europhys. Lett. 34575 (1996).

[41] H. Diamant, G. Ariel, and D. Andelman, Coll. Surf. A 183-185, 259 (2001).

[42] R. Podgornik, Chem. Phys. Lett. 163, 531 (1989).

[43] A. A. Kornyshev, D. A. Kossakowski, and S. Leikin, J. Chem. Phys. 97, 6809 (1992).

[44] R. Podgornik, and V. A. Parsegian, J. Phys. Chem. 99, 9491 (1995).

[45] H. N. Patrick, and G. G. Warr, J. Phys. Chem. 100, 16268 (1996).

[46] V. A. Parsegian, Trans. Faraday Soc. 62, 848 (1966).

[47] V. A. Parsegian, R. P. Rand, D. C. Rau, Method Enzymol. 259, 43 (1995).

[48] H. I. Petrache, S. Tristram-Nagle, K. Gawrisch, D. Harries, V. A. Parsegian, and J. F. Nagle, Biophys. J. 86, 1574 (2004).

[49] R. P. Rand, and V. A. Parsegian, Biochim. Biophys. Acta 988, 351 (1989).

[50] S. Leikin, V. A. Parsegian, D. C. Rau, and R. P. Rand, Annu. Rev. Phys. Chem. 44, 369 (1993).

[51] J. F. Nagle, and S. Tristram-Nagle, Biochim. Biophys. Acta Rev. 1469, 159 (2000).

[52] P. L. Hansen, R. Podgornik, and V. A. Parsegian, Phys. Rev. E. 64, 021907 (2001).

[53] P. Brocca, E. del Favero, and L. Cantu, personal communication.

[54] F. Ricoul, M. Dubois, L. Belloni, Th. Zemb, C. AndréBarrés, and I. Rico-Lattes, Langmuir 14, 2645 (1998).

[55] H. Boroudjerdi, Y.-W. Kim, A. Naji, R. R. Netz, X. Schlagberger, and A. Serr, Phys. Rep. 416, 129 (2005). 